\title{
Condition-based operational optimization of industrial combined heat and power plants under time-sensitive electricity prices
}

\author{
Egidio Leo, Sebastian Engell
}

Process Dynamics and Operations Group, Department of Biochemical and Chemical Engineering, Technische Universität Dortmund, EmilFigge-Str.70, 44221 Dortmund, Germany.

Increasing share of renewable energies, time-sensitive electricity prices and flexibility of the production resources have been identifying industrial Demand-Side Response (iDSR) as the main strategy to achieve significant reductions of operating costs for energy-intensive industries [1]. This strategy leads to strongly irregular and discontinuous operation of Combined Heat and Power (CHP) plant, in order to respect the user demand and maximize profits depending on the electricity price [2]. To avoid premature aging and unplanned maintenance costs due to failures of plant components, we propose a Mixed-Integer Linear Programming formulation aimed at maximizing the profit of a CHP under time-sensitive energy prices taking into account the Remaining Useful Life (RUL) of the plant. The CHP model is obtained adopting a mode formulation, able to account for different operating modes (cold-starts, warm-starts...) and reduce the computational effort. The RUL estimation is obtained from a fatigue crack growth model, a widely used prognostic model for mechanical systems with detectable cracks. However in this work the crack growth model is used to simulate the degradation process of the plant, proving a direct relation between plant load and plant degradation, and to predict its RUL: no crack measurement is needed. The objective function of the optimization problem takes into account the cost or the income from the electricity sold or purchase and the deprecation of the asset value due to the RUL reduction. It is worth to highlight that strong irregular operations, like shut-downs, are introduced into the objective function by the asset deprecation they produce for the RUL reduction and not with cost coefficients difficult to estimate. To study the advantages of the proposed framework, two optimized strategies are compared: the well-known iDSR and the proposed condition-based iDSR, aware of the equipment lifetime decrease. The results show that the standard iDSR approach is able to follow the electricity price generating electricity during hours of favourable prices, and timing the shutdowns and start-ups of generation assets, satisfying the steam and electricity demand of the chemical park. Shutdowns increase the plant profit since production is stopped and electricity is bought from the power grid. However degradation mechanics are not controlled and the lifetime deteriorates drastically. On the other hand, the condition-based optimization leads the system to reduce the number of shut-downs of critical components and the plant operation becomes less aggressive. Since the goal of power plant owners is to minimize plant operating costs, plant depreciation and degradation must be included in this computation.

[1] Merkert et al, Scheduling and energy-Industrial challenges and opportunities, Comput. Chem. Eng.

[2] Mitra et al, Optimal scheduling of industrial combined heat and power plants under time-sensitive electricity prices. Energy 2013 